\title{
A 64-lead Body Surface Potential Mapping System
}

\author{
João L Salinet ${ }^{1,2}$, Victor G Marques ${ }^{1,2}$, Marcelo Mazzetto ${ }^{2}$, Erick D L B Camargo ${ }^{1}$, Carlos A \\ Pastore $^{2}$, Idágene A Cestari ${ }^{2}$ \\ ${ }^{1}$ Federal University of ABC, São Bernardo do Campo, Brazil; \\ ${ }^{2}$ Heart Institute (InCor) - University of São Paulo, São Paulo, Brazil.
}

\begin{abstract}
Non-invasive acquisition of the electrical heart activity through high density mapping might allow early diagnosis of heart diseases overcoming the limitations of the traditional ECG method. This study presents a BSPM system (hardware and platform) to allow users to analyze the characteristics of morphology in up to 64 simultaneous body surface potentials (BSPs) including the 12-lead ECG and vectocardiogram (VCG). The signals undergo a preprocessing step followed by the R peak detection using previously validated techniques for heart rate variability studies. In addition, embedded $3 D$ isopotential, $3 D$ isochrone maps and VCG planes allow researchers to investigate the heart's the electrical activity and its patterns under different heart rhythm disorders in clinical practice.
\end{abstract}

\section{Introduction}

The Electrocardiographic (ECG) has been worldwideutilized as a clinical tool for diagnosis of the electrophysiological heart functions. Its 12 leads configuration (Fig.1a) comprises of bipolar limb leads (I, II and III), augmented unipolar limb leads (aVR, aVL and AVF) and unipolar chest leads (V1 to V6) allowing clinicians at identifying abnormalities of the heart's electrical activity [1]. The ECG's non invasive nature and low cost, the easy access and excellent reproducibility have contributed extensively to its popularity as a clinical tool [2]. The Vectocardiogram (VCG) has been used with the ECG and represents the heart's vector by its projections on three planes. This method has helped to supplement information not easily detectable through traditional electrocardiographic analysis [1]. From the several orthogonal corrected systems leads proposed, the Frank's method [3] has been the most popular protocol accepted by the scientific community, with 7 electrodes (Fig. 1b) to determine the horizontal (X), vertical (Y) and antero-posterior(Z) components. These components combined two by two give source to three orthogonal planes where will be projected the spacial curves representative from the electrical heart's phenomenon.
Although efforts have been made to improve the mechanism's understanding of heart disorders, the reduced number of electrodes (low spatial resolution) still the major limitation on identifying precise diagnostic of certain heart diseases $[1,4,5]$. A device containing a high-resolution of simultaneous non-invasive recordings would allow better detection of local variations in heart activity on the body surface potentials (BSPs), helping on the diagnosis of heart diseases [6] (Fig. 1c). A developed device called Body Surface Potential Mapping (BSPM) has been utilized in both research and clinical practices [7] allowing real-time acquisition of BSPs over a range of 32 to 300 electrodes providing better diagnostics of several heart disturbances $[1,4,5]$. Besides these advantages, the BSPM also allows investigators to map patterns of propagation through a three-dimensional (3D) colour-coded torso map of the electrical activity. Moreover, a convenient placement of the electrodes could provide information for the BSP potentials together with ECG waveforms and VCG plots. This study aims to develop a BSPM system with 64 electrodes to perform in real time, integrating the 12 lead ECG and VCG techniques.
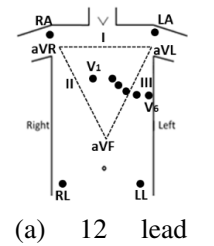
ECG

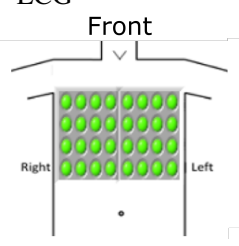

(c) BSPM (64 electrodes)

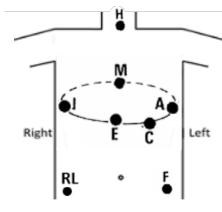

(b) Frank's VCG

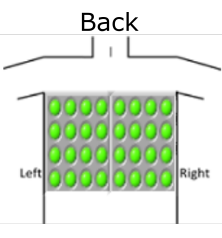

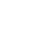

Figure 1: Lead positions on different electrical heart exams 


\section{Methods}

The present developed BSPM system consists of a dedicated hardware and a customized platform that runs on an executable file generated in LabVIEW ${ }^{\mathrm{TM}}$ (version 14.0.1f3, 32-bit). Fig. 2 provides an overview of the system's structure and features.

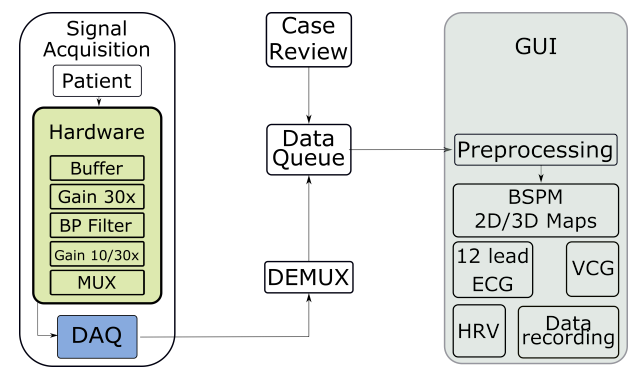

Figure 2: Block diagram of the system

\subsection{Simulated and experimental data}

BSPs from several sources were used to validate the proposed BSPM system. The simulated 12-lead ECG generated with a Bio-Tek 601 Pro safety analyzer was used to adjust the analogic gain and digital processing. VCG records obtained from a Physionet PTB databank [8] were used to validate the VCG plots. Patients who had BSPs recorded by a different BSPM system ( 2 healthy and 2 in atrial fibrillation) were exported to off-line analysis and uploaded into the proposed system in order to calibrate the 2D/3D maps. A 64-lead BSPM was recorded in a volunteer to assure the functionality of the system.

\subsection{Hardware}

The hardware is organized in 4 modules with 16 channels each, which perform analog amplification and filtering of the signal. Each channel has an overload protection circuit consisting of a neon lamp to absorb the discharge and Zener diodes to limit the input tension. The signals are then conducted to an input buffer for impedance matching and amplified 30 times ( $29.54 \mathrm{~dB}$ ), followed by bandpass filtering between the frequencies of 0.5 and $150 \mathrm{~Hz}$. A second amplifier with programmable gain of 10 or 30 times (totalizing either 49.54 or $59.08 \mathrm{~dB}$ amplification) is applied. Each module contains a sample hold circuit, a 16 channel multiplexer and a high tension isolating amplifier (rupture tension at $8 \mathrm{kV}$ ). A control board sends 16 $\mathrm{kHz}$ pulses to a National Instruments (NI) USB-6363 data acquisition system (DAQ), which performs the analog to digital (AD) conversion accordingly, providing digital signals with 16 bits of precision. Each module containing 16 multiplexed BSPM signals corresponds to a channel from the DAQ. The demultiplexation is made via software. The reference for all the channels is the right leg.

\subsection{Platform}

The BSPM platform contains a graphical user interface (GUI) divided in tabs, in which several features of the system are distributed. It also controls the source of the data, between two possible modes: patient examination and case review.

Preprocessing: The digitalized BSPs are demultiplexed in 64 channel with $1 \mathrm{kS} / \mathrm{s}$ sampling rate. The digital filters' specifications follow standard international recommendations [9]. A second order Savitzky-Golay filter with cutoff frequency at $150 \mathrm{~Hz}$ is used to remove high frequency components [10]. Notch filtering is performed in order to eliminate the $60 \mathrm{~Hz}$ powerline interference [11], with notch band of $6 \mathrm{~Hz}$ and passband attenuation of $-0.5 \mathrm{~dB}$. Baseline is removed by subtracting the output of a second order Lynn's lowpass filter from the delayed output [12], eliminating frequencies below $0.26 \mathrm{~Hz}$. Every filter has linear phase, essential to avoiding deformations in the waveform. Preprocessing occurs in pseudo real time configuration, with use of buffers.

Waveform visualization: The waveforms from each of the 64 BSPM leads can be individually analyzed, with the possibility of ploting up to 4 BSPs simultaneously. A global overview is also provided, in order to spot defective electrode connections and BSPs' abnormalities.

The traditional 12 leads of the ECG can be extracted from selected electrodes on the torso, in order to maintain the original direction of the measurement. Subtracting values from two such BSPM unipolar leads yields the differential leads as result (ie. I, II, III, aVL, aVR and aVF). The precordial leads (V1-6) are obtained directly from the system. The Pan-Tompkins algorithm is applied on a selected lead (DII default) in order to allow monitoring heart rate, VCG plots and heart rate variability (HRV) analysis.

The vectocardiograms are extracted based on Frank's lead system [3], where three orthogonal leads corresponding to the axis of the body ( $\mathrm{Vx}, \mathrm{Vy}$ and $\mathrm{Vz})$ are calculated (equations 1 to 3 ) based on potential values in predetermined torso's leads position (Fig. 1b) [1, chap. 11]. ECG leads V3 and V6 are common with electrodes $\mathrm{C}$ and $\mathrm{A}$ from the VCG, respectively.

$$
\begin{array}{r}
V_{x}=0.61 V_{A}+0.171 V_{C}-0.781 V_{I} \\
V_{y}=0.655 V_{F}+0.345 V_{M}-1.0 V_{H} \\
V_{z}=0.133 V_{A}+0.736 V_{M}-0.264 V_{I} \\
-0.374 V_{E}-0.231 V_{C}
\end{array}
$$

Potential maps: The body surface leads are obtained from the potentials measured in 64 electrodes on the torso, 
all of which use the Wilson's central terminal as a reference to the single-ended acquisition. A 3D torso geometry was used to plot potential values instantaneously; 2D maps were obtained by showing the measured values as a color coded matrix, according to the type of map selected.

A standardized 3D geometry of the torso was obtained from the online education and research ECGSIM software [13]. This torso has 410 vertices with their respective geometry (X, Y and Z) coordinates. The Matlab function 'patch' can build the 3D mesh by triangulating a sequence of three distinct vertices; this is done 816 times. Since there is no similar function in LabVIEW, 399 vertices of the torso have been selected (excluding neck and hip) and for each $\mathrm{X}, \mathrm{Y}$ and $\mathrm{Z}$ coordinates a corresponding matrices of dimensions $21 \times 19$ was produced. The standardized 3D Torso is created by the "3D Surface plot" function. To generate the sequential 3D BSPM, a fourth matrix (21x19) is utilized and the positions of the correspondent leads are identified. The recorded electrical activity is then color coded, with blue representing lower voltages and red-dark corresponding to higher potentials; this is simultaneously shown as a $2 \mathrm{D}$ matrix.

In order to perform further analysis on the BSPs, the user can select a 2 seconds segment and, with the help of a cursor, investigate sequential maps and their respective 64 BSPs. Isopotential or isochrone maps can be viewed to allow patterns' investigation of the potentials on the torso and depict activation sequences over the heart cycle. The $\mathrm{R}$ peak detection is done by the Pan-Tompkins QRS detection algorithm [14]. Both maps can be displayed in 2D or 3D configuration.

The individual leads ( $\mathrm{Vx}, \mathrm{Vy}$ and $\mathrm{Vz}$ ) and the spatial VCG's projection on the three planes of the body (X, horizontal, Y, vertical Y and Z, antero-posterior) representing the electrical heart's cycle phenomenon are presented on the platform. A fixed time window around a detected $\mathrm{R}$ peak is used to define P-QRS-T boundaries for the VCG.

\section{Results}

Figure 3 illustrates the BSPM platform. A standardized 3D geometry of the torso is presented and colour coded according to the electrical potentials in 64 four distinct regions, allowing investigators to identify patterns of heart electrical activation and its wavefront propagation noninvasively. The electrodes are equally distributed on both front and back, in sets with 32 electrodes each (4 strips of 8 electrodes) and users can define the electrodes setup of the research protocol by checking on the specific electrodes to register the torso's electrical activity. The system operates in monitoring mode where no record is done for off-line analysis until users' decision. The platform allows users to visualize both raw and preprocessed BSPs. The preprocessing filters and their cut-off frequencies are set with default values, but can be modified when necessary.

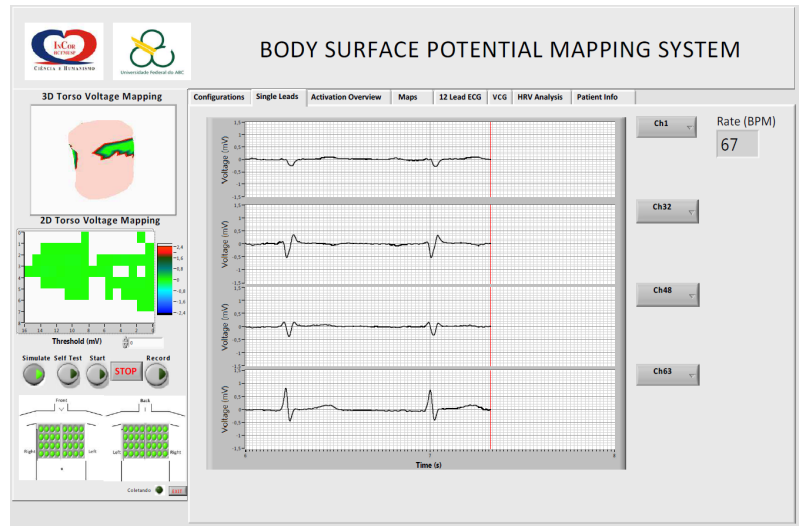

Figure 3: BSPM platform

Investigation of the BSPs characteristics such activations' dynamics and morphology presented within R-R intervals are accessible in all BSPs, with up to four selected for simultaneous visualization. If users' option is to concentrate on the P-QRS-T complexes, the BSPM Activation Overview tab shows the respective individual complexes of all electrodes (see Fig. 4). The 12 lead ECG and the VCG plots are presented on specifics tab for user analysis (see Fig. 6 and 7). Instantaneous Heart Rate (mean \pm std), QRS complex amplitude (mean \pm std), R-R interval (mean \pm std) and others HRV time analyses metrics are also available to the user. In Fig. 5 the display type (isopotential or isochrone) is defined by the user and a cursor selects the instant on the heart cycle shown in the maps.

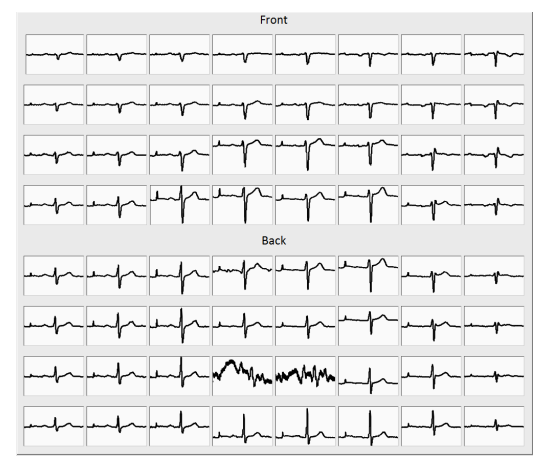

Figure 4: "Activation Overview" tab

\section{Discussion and conclusion}

We have developed a real time BSPM system with 64 channels for investigation of heart's the electrical activity and its patterns under different heart rhythm disorders in clinical practice. The systems allows visualization of BSPs waveforms, 3D isopotential and isochrone maps and 


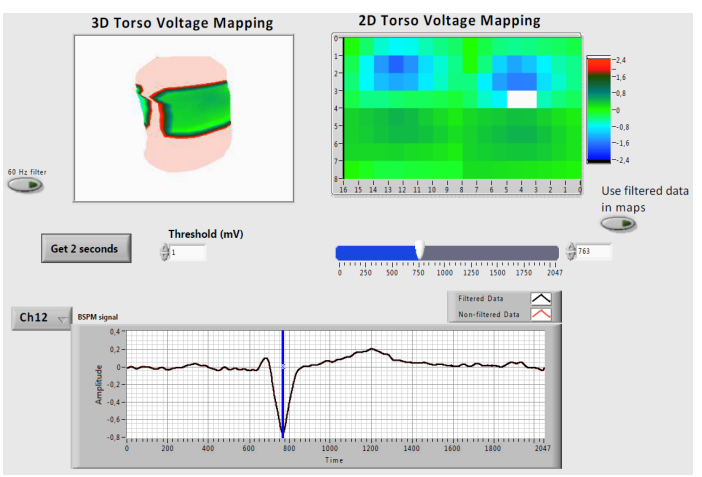

Figure 5: Detailed view of the maps

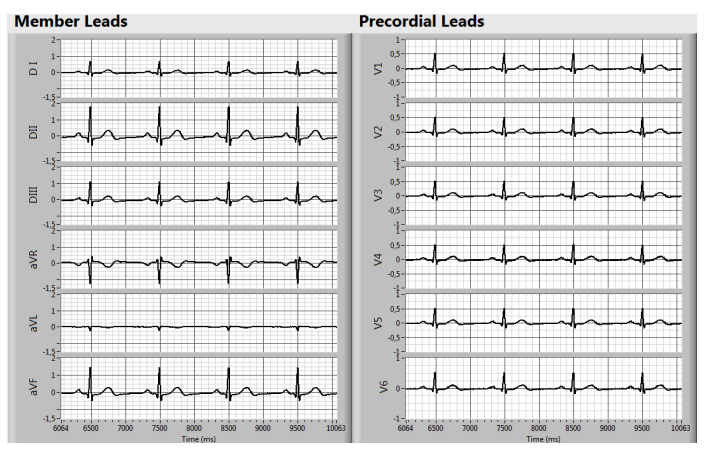

Figure 6: 12 lead ECG signals

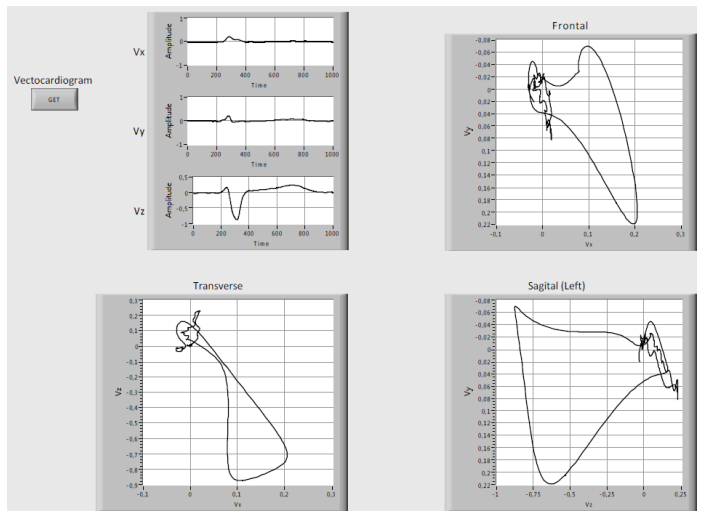

Figure 7: Frank lead VCG - single leads and projections

the spacial electrical heart's phenomenon projection (VCG plots). Fast acquisition and close to real time processing of the signal allow the system to display quality plots instantaneously to clinicians and researchers alike, configuring a powerful tool for non invasive heart disease diagnosis.

\section{Acknowledgements}

The authors would like to thank Dr. Fernando Schlindwein and Prof G André Ng from University of Leicester for providing body surface potential signals. This work was supported by São Paulo Research Foundation (FAPESP Grants N 2016/26240-6 and 2017/11103-6).

\section{References}

[1] Macfarlane PW, Van Oosterom A, Pahlm O, Kligfield P, Janse M, Camm J. Comprehensive electrocardiology. Springer Science \& Business Media, 2010.

[2] Bronzino JD. Biomedical engineering handbook, volume 2. CRC press, 1999.

[3] Frank E. An accurate, clinically practical system for spatial vectorcardiography. Circulation 1956;13(5):737-749.

[4] Taccardi B, Punske BB, Lux RL, MacLEOD RS, Ershler PR, Dustman TJ, Vyhmeister Y. Useful lessons from body surface mapping. Journal of Cardiovascular Electrophysiology 1998;9(7):773-786.

[5] Medvegy M, Duray G, Pintér A, Préda I. Body surface potential mapping: Historical background, present possibilities, diagnostic challenges. Annals of Noninvasive Electrocardiology 2002;7(2):139-151.

[6] Graaf A, Bhagirath P, Ramanna H, Driel VJ, Hooge J, Groot N, Götte MJ. Noninvasive imaging of cardiac excitation: current status and future perspective. Annals of Noninvasive Electrocardiology 2014;19(2):105-113.

[7] Ramanathan C, Ghanem RN, Jia P, Ryu K, Rudy Y. Noninvasive electrocardiographic imaging for cardiac electrophysiology and arrhythmia. Nature medicine 2004; 10(4):422-428.

[8] Goldberger AL, Amaral LA, Glass L, Hausdorff JM, Ivanov PC, Mark RG, Mietus JE, Moody GB, Peng CK, Stanley HE. Physiobank, physiotoolkit, and physionet: Components of a new research resource for complex physiologic signals. Circulation 2000;101(23):e215-e220.

[9] Kligfield P, Gettes LS, Bailey JJ, Childers R, Deal BJ, Hancock EW, van Herpen G, Kors JA, Macfarlane P, Mirvis $\mathrm{DM}$, et al. Recommendations for the standardization and interpretation of the electrocardiogram. Circulation 2007; 115(10):1306-1324.

[10] Christov I, Neycheva T, Schmid R, Stoyanov T, Abächerli R. Pseudo-real-time low-pass filter in ecg, self-adjustable to the frequency spectra of the waves. Medical Biological Engineering Computing 2017;1-10.

[11] Zahradnik P. Notch filtering suitable for real time removal of power line interference. Radioengineering 2013; 22(1):186-193

[12] Kozumplík J, Provazník I. Fast time-varying linear filters for suppression of baseline drift in electrocardiographic signals. Biomedical engineering online 2017;16(1):24.

[13] Van Oosterom A, Oostendorp T. Ecgsim: an interactive tool for studying the genesis of qrst waveforms. Heart 2004; 90(2):165-168.

[14] Pan J, Tompkins WJ. A real-time qrs detection algorithm. IEEE transactions on biomedical engineering 1985; 32(3):230-236.

Address for correspondence:

Dr João Salinet (joao.salinet@ufabc.edu.br)

CECS - Federal University of ABC (UFABC) 